Case Report

\title{
Refractory Anemia with Ring Sideroblasts Associated with Marked Thrombocytosis Complicated by Massive Splenomegaly Treated with Lenalidomide Resulting in Resolution of Splenomegaly but Severe and Prolonged Pancytopenia
}

\author{
Gordon Taylor, Dominic Culligan, and Mark A. Vickers \\ Department of Haematology, Aberdeen Royal Infirmary, Aberdeen AB25 2ZN, UK \\ Correspondence should be addressed to Gordon Taylor; gordon.taylor1@nhs.net
}

Received 4 February 2013; Accepted 28 February 2013

Academic Editors: E. Bissé, K. Kawauchi, R. Tiu, and P. Tsirigotis

Copyright (c) 2013 Gordon Taylor et al. This is an open access article distributed under the Creative Commons Attribution License, which permits unrestricted use, distribution, and reproduction in any medium, provided the original work is properly cited.

Refractory anemia with ring sideroblasts associated with marked thrombocytosis (RARS-T) is a hematological malignancy that combines features of both a myeloproliferative and myelodysplastic disorder. There have been recent reports of the successful treatment of anemia in 2 patients with RARS-T with lenalidomide. Here we report the successful treatment of massive splenomegaly in a patient with a long history of RARS-T resulting in complete resolution of splenomegaly, but with prolonged severe cytopenias. We also report the acquisition of the $\mathrm{t}(3 ; 12)(\mathrm{q} 26 ; \mathrm{p} 13)$ translocation previously described in cases of myelodysplasia and the potential for transformation to myelofibrosis.

\section{Case Presentation}

A 47-year-old female presented 12 years ago with migrianous headaches, a persistent marked thrombocytosis (greater than $700 \times 10^{9} / \mathrm{L}$ ), and $25 \%$ bone marrow ring sideroblasts. Bone marrow cytogenetics showed a normal 46XX karyotype. The subsequent development of anemia with dyserythropoiesis, increasing percentage of ring sideroblasts to $45 \%$, and confirmation of the presence of the JAK2V617F mutation allowed the diagnosis to be confirmed as the recently characterised refractory anemia with ring sideroblasts associated with marked thrombocytosis (RARS-T). With disease progression she developed massive splenomegaly extending past her umbilicus. Splenomegaly was unsuccessfully treated with hydroxycarbamide, since doses large enough to cause a minor reduction in spleen size resulted in worsening of anemia requiring red cell transfusion. Following the report during 2010 of two recently diagnosed patients with RARS-T showing an improvement in anemia when treated with lenalidomide, treatment was changed to lenalidomide $10 \mathrm{mg}$ daily, taken for 21 days out of every 28 day cycle. Within 2 weeks of starting lenalidomide the patient became pancytopenic and her transfusion requirement increased. After completing a total of 4 monthly cycles of lenalidomide, she remained heavily transfusion dependent (requiring 2 to 3 units of red cells every 1-2 weeks) and had persistent National Cancer Institute common toxicity criteria grade 4 neutropenia and grades 2-3 thrombocytopenia. Lenalidomide treatment was therefore stopped. During the treatment period her spleen had started to soften and over the following 2 months off treatment there was complete resolution of her palpable massive splenomegaly. She remained profoundly pancytopenic and red cell transfusion dependent (Figure 1).

A bone marrow biopsy sample was taken 6 weeks after stopping lenalidomide. The trephine biopsy revealed clusters of dysplastic megakaryocytes and erythroid cells as before, but now with frank marrow fibrosis (grade 3 ) that had not been present at diagnosis (Figures 2(a) and 2(b)).

Four months after stopping lenalidomide her spleen had grown back to past her umbilicus, her transfusion frequency was reduced, her neutrophil count had recovered to greater than $1 \times 10^{9} / \mathrm{L}$, and her platelet count had returned to within normal range. The patient underwent an unrelated donor hematopoietic stem cell transplant using reduced intensity 


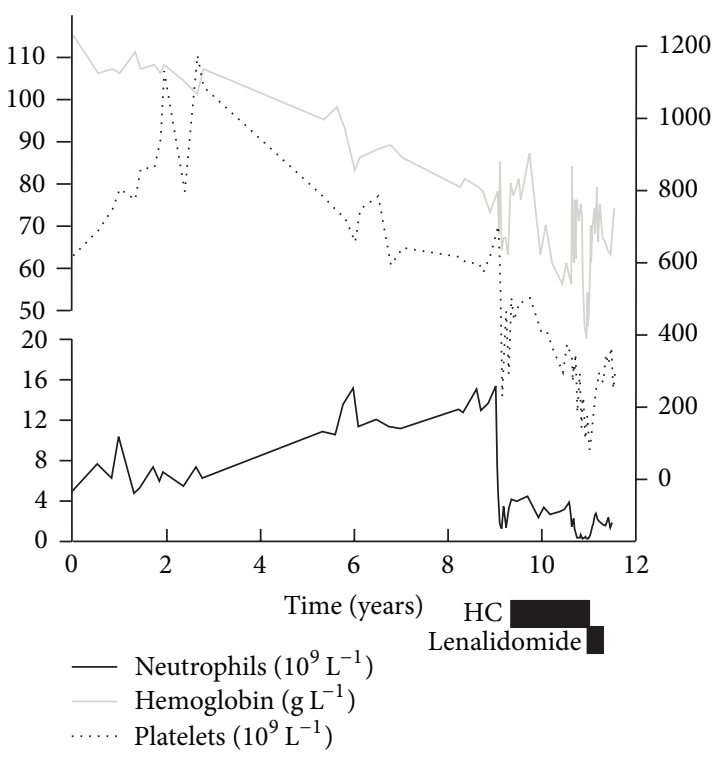

Figure 1: Table demonstrating neutrophil count, platelet count, and pretransfusion hemoglobin against time from diagnosis up until the time of transplant, also showing the effect of hydroxycarbamide and lenalidomide on these.

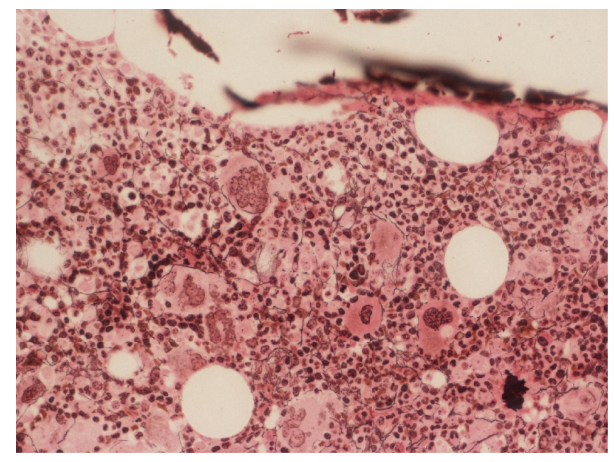

(a)

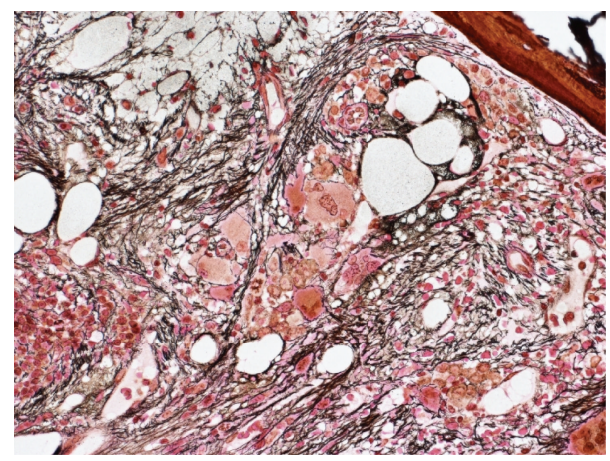

(b)

FIGURE 2: (a) demonstrates the patient's bone marrow reticulin stain at initial presentation demonstrating only fine reticulin deposition around megakaryocytes. (b) is bone marrow biopsy 10 years after diagnosis demonstrating grade 3 reticulin fibrosis. conditioning of fludarabine, melphalan, and alemtuzumab. Following an immediate posttransplant period of poor engraftment, with donor chimerism of not more than $30 \%$, the graft was lost with progression of massive splenomegaly and increasing transfusion dependence. Repeat bone marrow biopsy 125 days posttransplant identified the presence of a t $(3 ; 12)$ (q26;p13) translocation involving ETV6 and EVI1, not previously identified in this patient. This translocation has previously been described in cases of acute myeloid leukemia, myelodysplastic syndromes (MDS), and chronic myeloid leukemia. The patient received splenic radiotherapy for control of pain and started treatment with the combined $J a k l$ and Jak2 inhibitor ruxolitinib but did not respond to this and subsequently died from sepsis.

\section{Discussion}

RARS-T is a provisional World Health Organization recognised disease entity amongst the group of myeloproliferative/myelodysplastic crossover syndromes [1] and is associated with the presence of the Jak2V617F mutation [2], Tet2 mutations, and less commonly the MPLW515K/L mutation [3]. These mutations probably contribute to the myeloproliferative component of the disease. Recently described SF3B1 mutations appear to be associated with myeloid neoplasms with ring sideroblasts $[4,5]$. Lenalidomide is an immunomodulatory thalidomide analogue (IMiD) that has been shown to have some efficacy in the treatment of anemia in other myelodysplastic syndromes [6,7], particularly when associated with deletions of chromosome $5 \mathrm{q}$, where transfusion independence occurs in some two-thirds of patients [7]. Lenalidomide with prednisolone has also been reported to be efficacious in improving anaemia and splenomegaly in idiopathic myelofibrosis although treatment has been limited by high rates of grades 3-4 hematological toxicity [8].

The two original cases reported by Huls et al. [9] detailing improvements in anemia in patients with RARS-T treated with lenalidomide, importantly, did not appear to have had significant marrow fibrosis at the time of initiation of lenalidomide therapy. In our case, described here, lenalidomide was used at an advanced stage of the disease, when there was clearly extensive marrow fibrosis and massive splenomegaly. The response to lenalidomide in this situation was interesting in that there was complete resolution of the massive splenomegaly but profound worsening of the cytopenias. The likely explanation for this is that the extramedullary hematopoiesis in the spleen is predominantly arising from the malignant JAK2V617F mutated clone. Lenalidomideinduced suppression of the clone led to effective resolution of splenomegaly but there is insufficient normal residual hematopoiesis in the fibrotic marrow to allow for any hematopoietic recovery at this late stage in the natural history of RARS-T. In many ways this is akin to the myelosuppression that predicts response to lenalidomide therapy in MDS with del $5 \mathrm{q}$ and is thought to result from direct suppression of the $5 \mathrm{q}$-clone but in this instance occurs in the context of sufficient functional hematopoiesis to allow for blood count recovery. A reduction in the detectable JAK2V617F-positive malignant 
clone was also reported in the 2 cases of RARS-T treated with lenalidomide by Huls et al. [9].

Thalidomide with and without prednisolone has been reported to produce responses in primary and secondary MF with improvement in anemia and splenomegaly; however long term treatment is often limited by significant rates of peripheral neuropathy [10]. Pomalidomide is a newer IMiD that does not appear to cause peripheral neuropathy and appears to cause less hematological toxicity than lenalidomide. There are reports of this being effective in the treatment of anemia and splenomegaly in patients with MF [11]. At the time of writing pomalidomide is not licensed for use in the UK. Although combined Jakl and Jak2 inhibitors appear to be effective in reducing spleen size and treating systemic symptoms in patients with primary MF and secondary MF, they appear to cause significant hematological toxicity, particularly worsening of anemia in around a quarter to a third of patients treated [12].

\section{Conclusion}

In conclusion, we describe the natural history of a young woman with RARS-T over nearly 12 years and confirm that marked marrow fibrosis and splenomegaly can occur. The pattern of response to lenalidomide should make physicians cautious when considering treatment with IMiDS in patients with RARS-T at an advanced stage of the disease, especially if there is significant bone marrow fibrosis.

\section{Conflict of Interests}

G. Taylor reports no conflict of interests. D. Culligan has received honoraria from Celgene for advisory boards and lectures. M. Vickers reports no conflict of interests.

\section{References}

[1] J. W. Vardiman, J. M. Bennet, B. J. Bain, I. Bauman, J. Thiele, and A. Orazi, "Myelodysplastic/myeloproliferative neoplasms, unclassifiable," WHO Classification of Tumours of Haematopoietic and Lymphoid Tissues, pp. 85-86, 2008.

[2] S. A. Wang, R. P. Hasserjian, J. M. Loew et al., "Refractory anemia with ringed sideroblasts associated with marked thrombocytosis harbors JAK2 mutation and shows overlapping myeloproliferative and myelodysplastic features," Leukemia, vol. 20, no. 9, pp. 1641-1644, 2006.

[3] J. Flach, F. Dicker, S. Schnittger, A. Kohlmann, T. Haferlach, and C. Haferlach, "Mutations of JAK2 and TET2, but not CBL are detectable in a high portion of patients with refractory anemia with ring sideroblasts and thrombocytosis," Haematologica, vol. 95, no. 3, pp. 518-519, 2010.

[4] M. M. Patnaik, T. L. Lasho, J. M. Hodnefield et al., "SF3B1 mutations are prevalent in myelodysplastic syndromes with ring sideroblasts but do not hold independent prognostic value," Blood, vol. 119, no. 2, pp. 569-572, 2012.

[5] V. Visconte, H. Makishima, A. Jankowska et al., "SF3B1, a splicing factor is frequently mutated in refractory anemia with ring sideroblasts," Leukemia, vol. 26, no. 3, pp. 542-545, 2012.
[6] A. List, S. Kurtin, D. J. Roe et al., "Efficacy of lenalidomide in myelodysplastic syndromes," New England Journal of Medicine, vol. 352, no. 6, pp. 549-557, 2005.

[7] A. List, G. Dewald, J. Bennett et al., "Lenalidomide in the myelodysplastic syndrome with chromosome 5q deletion," New England Journal of Medicine, vol. 355, no. 14, pp. 1456-1465, 2006.

[8] R. A. Mesa, X. Yao, L. D. Cripe et al., "Lenalidomide and prednisone for myelofibrosis: Eastern Cooperative Oncology Group (ECOG) phase 2 trial E4903," Blood, vol. 116, no. 22, pp. 4436-4438, 2010.

[9] G. Huls, A. B. Mulder, S. Rosati, A. A. Van De Loosdrecht, E. Vellenga, and J. T. M. De Wolf, "Efficacy of single-agent lenalidomide in patients with JAK2 (V617F) mutated refractory anemia with ring sideroblasts and thrombocytosis," Blood, vol. 116, no. 2, pp. 180-182, 2010.

[10] M. A. Elliott, R. A. Mesa, C. Y. Li et al., "Thalidomide treatment in myelofibrosis with myeloid metaplasia," British Journal of Haematology, vol. 117, no. 2, pp. 288-296, 2002.

[11] A. Tefferi, S. Verstovsek, G. Barosi et al., "Pomalidomide is active in the treatment of anemia associated with myelofibrosis," Journal of Clinical Oncology, vol. 27, no. 27, pp. 4563-4569, 2009.

[12] S. Verstovsek, H. Kantarjian, R. A. Mesa et al., "Safety and efficacy of INCB018424, a JAK1 and JAK2 inhibitor, in myelofibrosis," New England Journal of Medicine, vol. 363, no. 12, pp. 1117-1127, 2010. 


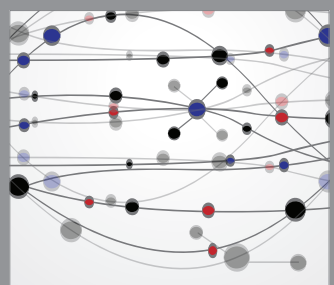

The Scientific World Journal
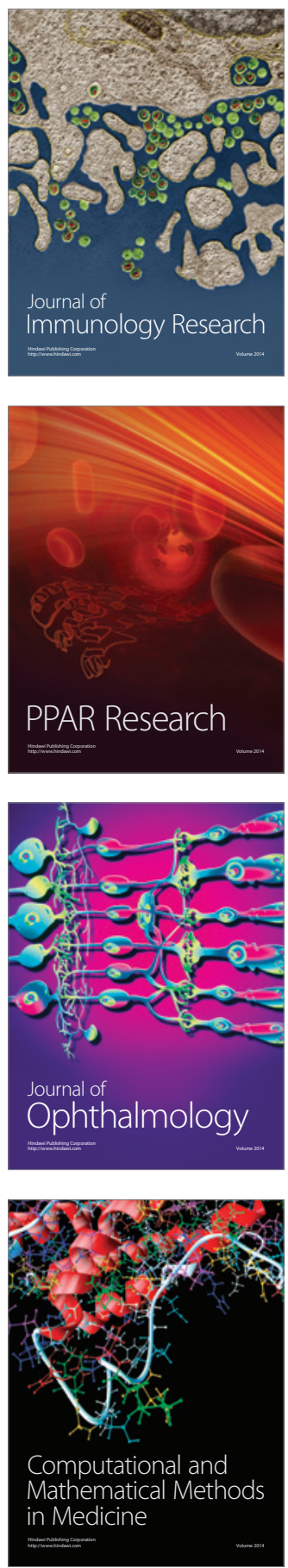

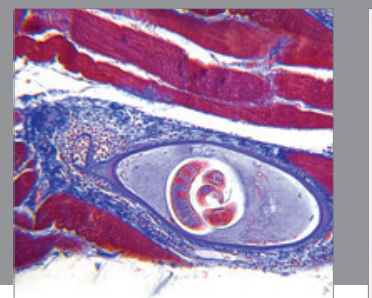

Gastroenterology

Research and Practice
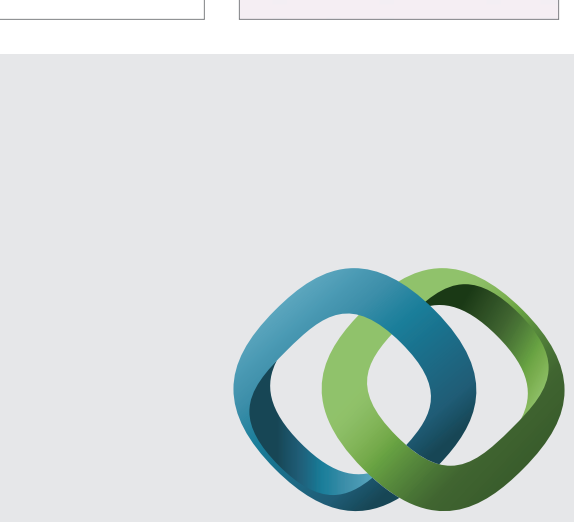

\section{Hindawi}

Submit your manuscripts at

http://www.hindawi.com
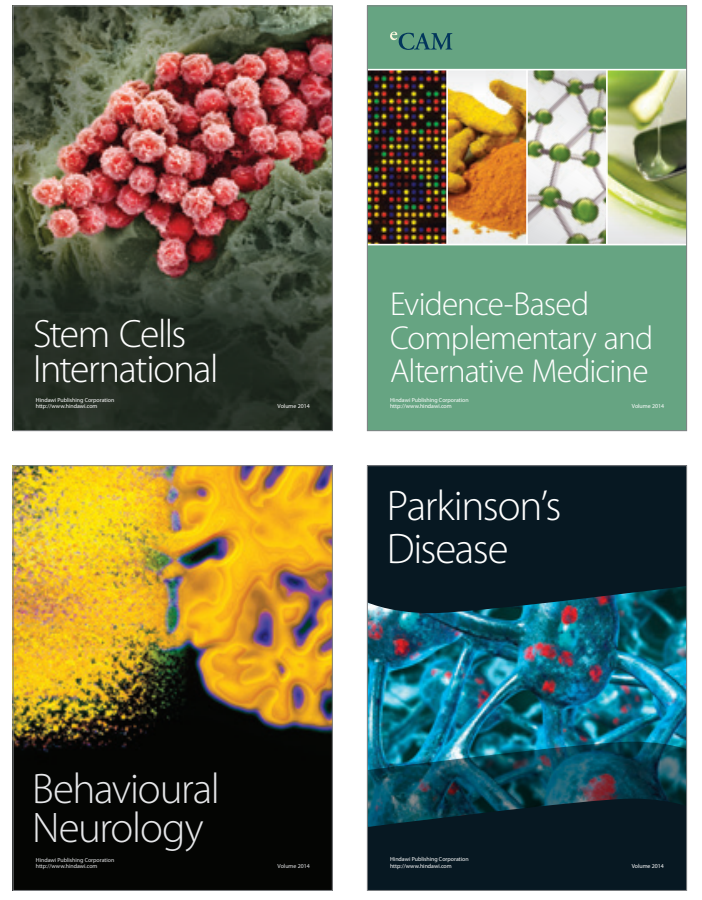
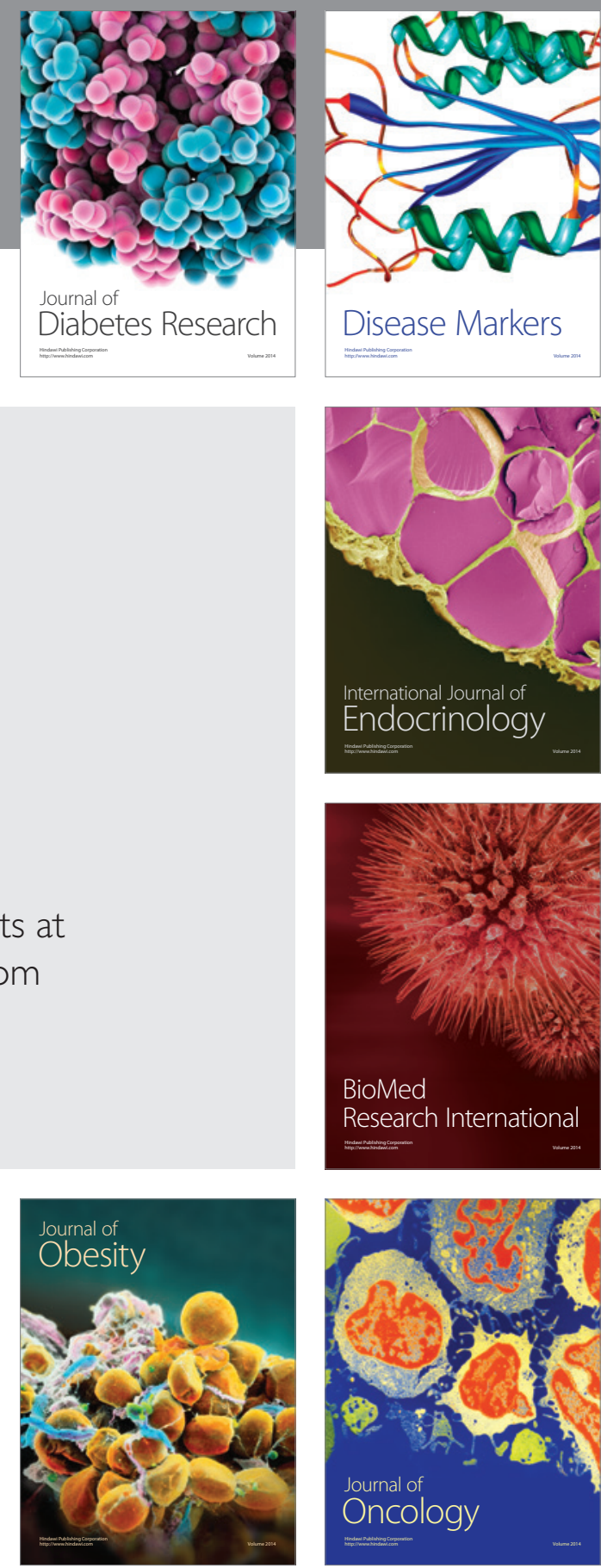

Disease Markers
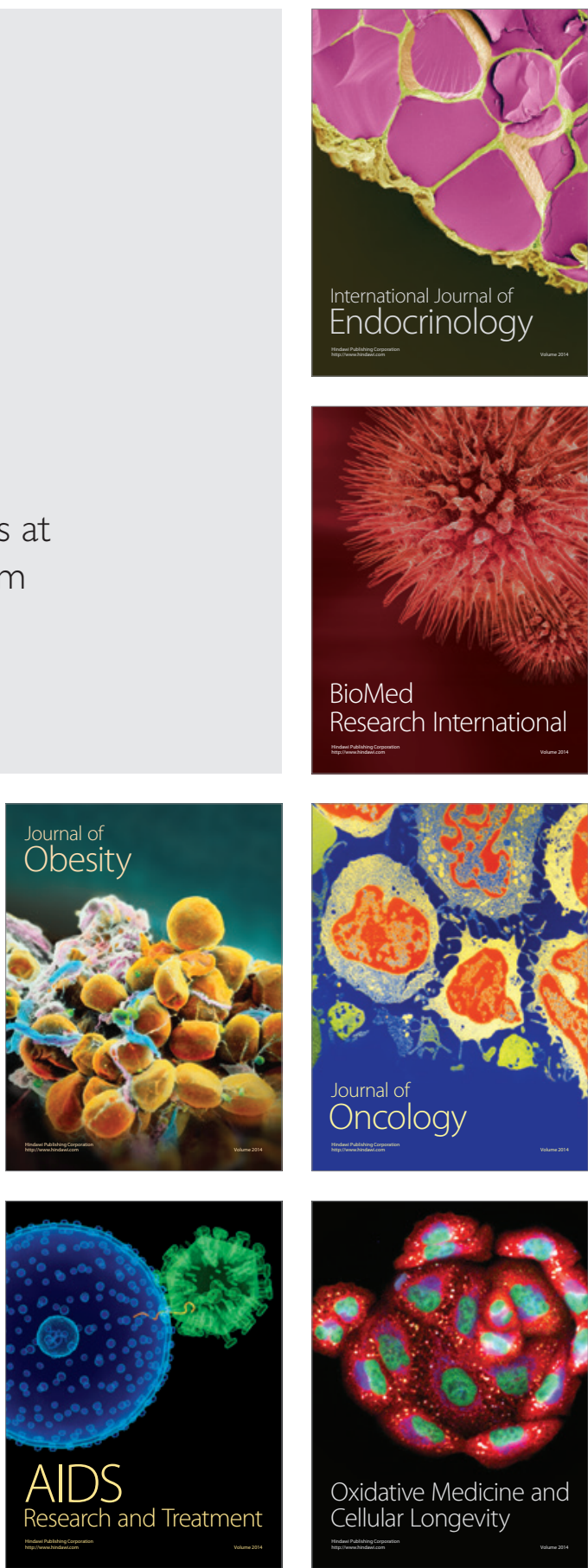